\title{
Reseña del libro "Análisis de los dispositivos de formación en la Universidad Pública Mexicana”
}

Elizabeth Zanatta Colín, Sergio Luis García Iturriaga, José Luis Gama Vilchis, Claudia Angélica Sánchez Calderón, Teresa Ponce Dávalos ((2014). Análisis de los dispositivos de formación en la universidad pública mexicana. Toluca: Universidad Autónoma del Estado de México.

A lo largo de los cinco capítulos que conforman este libro se realizan análisis muy interesantes sobre varios elementos de los modelos educativos de universidades públicas pertenecientes al Consorcio de Universidades Mexicanas (CUMex).

En forma concreta se analizan el sentido que guardan las directrices estipuladas en los modelos educativos de las universidades pertenecientes a CUMex, sus implicaciones para la formación universitaria y su pertinencia para favorecer la identidad institucional; toda vez que un modelo se sustenta en conceptos y premisas teóricas de los enfoques que comprenden un cierto sentido filosófico acerca de la educación.

Se habla en un primer momento de las tendencias curriculares en las universidades haciendo una reflexión de los principios que sustentan cada diseño curricular y las dimensiones de su práctica educativa tomando en cuenta la visión psicopedagógica, filosófica y sociológica. Se retoma el modelo de competencias que se instrumentó en algunas universidades del país; los primeros se caracterizan por procesos y objetivos simples, relativos a un sentido instruccional, siendo los modelos recientes los que integran una mayor complejidad.

Seguido a ello, se realza la importancia de la educación en valores ya que en la actualidad es algo que no puede sustraerse al contexto de la globalización. Se plantea a la educación como el medio y fin principal para la atención de los problemas sociales que se enfrentan día a día tales como actos de corrupción e impunidad por mencionar algunos. Así mismo, se hace una revisión de los fundamentos de los valores consignados en los documentos fuentes de las Universidades Públicas; de ello se toma en consideración la misión, la visión, los objetivos generales, los 
perfiles de ingreso y egreso, por mencionar algunos; identificando valores tanto personales, sociales, intelectuales y profesionales en estudiantes y profesores.

Más adelante, se explora sobre las políticas públicas que han impactado en la formación de las instituciones de educación superior, reflexionando acerca de si la decisión de convertirse en instituciones con calidad parte de los programas institucionales, de las prioridades del contexto que rodea a cada institución o, son fuerzas externas a las mismas instituciones las que presionan y condicionan el quehacer de la mayoría de las universidades publicas mexicanas. También se hace una reflexión sobre el concepto de calidad para poder llegar a consensos cuando se habla de ésta, y a través de una propuesta metodológica se buscaron modelos que permitan acuerdos que se refieran a los objetivos y procesos que favorezcan la adjetivación de programas académicos de "buena calidad" y así pertenecer al CuMex.

En el cuarto capítulo los autores realzan la importancia del conocimiento de los modelos de gestión que tienen las universidades para así tener un referente que sirva como guía en los procesos permanentes de mejora, en la formación del recurso humano, la generación de conocimientos y la difusión y extensión de la cultura. La gestión constituye la mejor forma de dirigir las instituciones educativas, las 27 universidades públicas que integran el CUMex conforman un espacio común para ofrecer programas de educación superior de calidad, por lo que resulta pertinente revisar los modelos educativos para detectar los cambios que han realizado en la gestión institucional, con el fin de lograr mayor eficiencia y eficacia en el desarrollo de sus funciones sustantivas y el logro de sus objetivos.

Por último, se reflexiona sobre la incorporación de las Tecnologías de Información y Comunicación (TIC) a las universidades considerándolas como un instrumento útil que favorece el desarrollo y fortalecimiento de las diferentes capacidades de los estudiantes, como un instrumento para la gestión en las instituciones y como apoyo para la evaluación institucional. Para que el aprovechamiento de las tecnologías sea eficaz, se requiere del despliegue de nuevas estrategias y metodologías pedagógicas implementadas por los docentes. En éste ámbito, la actitud de los docentes hacia los recursos tecnológicos y el cambio de sus prácticas educativas es decisivo para el impacto del proceso educativo. 
Como se alcanza a apreciar, las investigaciones que muestran los autores de este libro son temas de alta importancia para la formación en las universidades públicas mexicanas; e invitan a hacer una reflexión sobre el papel que juegan tanto docentes, académicos y estudiantes para un mejor futuro como sociedad.

Psic. Jessica Paola Martínez Orozco Universidad Autónoma del Estado de México 